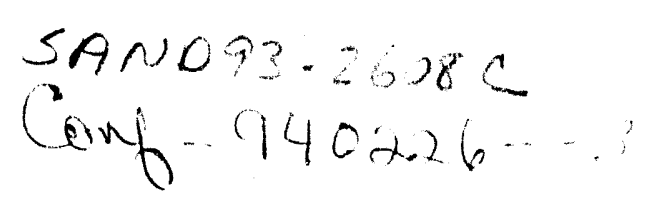

\title{
Characterization of a Ring Optical Parametric Oscillator
}

\author{
W.J. Alford, T.D. Raymond and A.V. Smith \\ Sandia National Laboratories \\ Albuquerque, NM 87185-5800 \\ (505)844-1244
}

\begin{abstract}
We have characterized the performance, including the spatial intensity profiles of the signal and depleted pump beams, of a near-infrared, ring optical parametric oscillator.
\end{abstract}

This work was supported by the United

States Department of Energy under

Contract DE-ACn4-94AL850no.

\section{DISCLAIMER}

\begin{abstract}
This report was prepared as an account of work sponsored by an agency of the United States Government. Neither the United States Government nor any agency thereof, nor any of their employees, makes any warranty, express or implied, or assumes any legal liability or responsibility for the accuracy, completeness, or usefulness of any information, apparatus, product, or process disclosed, or represents that its use would not infringe privately owned rights. Reference herein to any specific commercial product, process, or service by trade name, trademark, manufacturer, or otherwise does not necessarily constitute or imply its endorsement, recommendation, or favoring by the United States Government or any agency thereof. The views and opinions of authors expressed herein do not necessarily state or reflect those of the United States Government or any agency thereof.
\end{abstract}

\section{Masitin}




\title{
Characterization of a Ring Optical Parametric Oscillator
}

\author{
W.J. Alford, T.D. Raymond and A.V. Smith \\ Sandia National Laboratories \\ Albuquerque, NM 87185-5800
}

(505)844-1244

Optical parametric oscillators (OPOs) are capable of producing widely tunable coherent light using a fixed wavelength laser as a pump source. Though OPOs were first demonstrated in the 1960's, OPOs have recently been the subject of much research and development. The two principle reasons for this renewed interest have been the development of improved pump lasers, e.g. injection seeded Nd:YAG lasers, and improved nonlinear crystals, e.g. beta-barium borate (BBO), potassium titanyl phosphate (KTP), and lithium triborate (LBO). Despite the demonstration of OPOs using a large number of nonlinear crystals and cavity configurations, little work has been devoted to carefully characterizing the output energy and the temporal and spatial intensity profiles for well defined pump and cavity conditions. Such measurements are necessary for understanding the limitations and potential of OPOs as convenient sources of tunable coherent light and to advance the state-of-theart in computer simulations of these devices.

We have studied a singly-resonant KTP ring OPO pumped by nanosecond pulses from a frequencydoubled Nd:YAG laser. We present measurements of the temporal and spatial intensity profiles of the incident pump beam and OPO output beams, including the depleted pump, as well as the output energy as a function of pump laser energy. These measurements have been carried out for both injection-seeded and unseeded operation of the OPO. Thus we have carefully characterized the performance of a simple ring OPO.

The experimental setup is shown in Figure 1. The $1064 \mathrm{~nm}$ output from the oscillator of a Q-switched, injection-seeded Nd:YAG laser (Continuum NY61) is sent through a $2 \mathrm{~m}$ focal length lens and noncritically frequency doubled in a $2 \mathrm{~cm} \mathrm{LBO}$ crystal $(x$-cut) placed $60 \mathrm{~cm}$ from the lens. The YAG laser oscillator is capable of producing $150 \mathrm{~mJ}$ of $1064 \mathrm{~nm}$ at the optimum laser Q-switch delay. However, we typically lengthen the Qswitch delay to produce $<100 \mathrm{~mJ}$ in a beam with better beam quality than that produced at optimum delay. After separating the $532 \mathrm{~nm}$ light from the $1064 \mathrm{~nm}$ light the $532 \mathrm{~nm}$ light is focused onto a $400 \mu$ diamond pinhole to spatially filter the $532 \mathrm{~nm}$ beam. A collimating telescope is then used to give a $.6 \mathrm{~mm}$ full width at half maximum (FWHM) $532 \mathrm{~nm}$ beam with an energy of up to about $15 \mathrm{~mJ}$ in a $8 \mathrm{nsec}$ FWHM pulse. The 532nm pump beam spatial profile is well approximated by a Gaussian intensity distribution. Pump fluences of up to $2.5 \mathrm{~J} / \mathrm{cm}^{2}$ are used to pump the OPO.

The OPO is a three-mirror (flat-flat-flat) ring configuration similar to that used by Hamilton and Bosenberg ${ }^{1}$ consisting of two $780 \mathrm{~nm}$ high reflectors and a $780 \mathrm{~nm} 51 \%$ output coupler. One of the high reflectors is mounted on a piezoelectric transducer to allow finc adjustments of the cavity length. The KTP crystal $\left(\theta=51^{\circ}\right.$, $\left.\phi=0^{\circ}\right)$ is $1 \mathrm{~cm}$ in length with anti reflection coatings for $780 \mathrm{~nm}$ and $532 \mathrm{~nm}$. The OPO cavity length is $6.7 \mathrm{~cm}$. The resonated signal wave at $780 \mathrm{~nm}$ is polarized in the plane of the ring cavity and propagates through the KTP as an extraordinary wave. The pump and idler waves are polarized out of the plane of the ring and propagate through the KTP as ordinary waves. As the $780 \mathrm{~nm}$ wave traverses the crystal, it experiences a walkoff of $.52 \mathrm{~mm}$ in the plane of the resonator. A CW Ti:sapphire laser pumped by an $\mathrm{Ar}^{+}$ion laser (Spectra Physics 2020) can be used to injection seed the OPO. Typically the seed beam contains $30-40 \mathrm{~mW}$ of power in a beam slightly larger than the $532 \mathrm{~nm}$ pump beam.

The depleted pump and signal beams exiting the OPO are monitored with CCD cameras (Cohu 4800) and a fast phototube (Hamamatsu 1328U). The cameras are part of a Big Sky Aralyzer Plus beam profiler system. The OPO signal energy is measured with a pryoclectric detector (Laser Precision Rj-7200).

Figure 2 shows the OPO signal energy versus $532 \mathrm{~nm}$ pump energy. Threshold is observed to be at a pump energy of $3.6 \mathrm{~mJ}(5.2 \mathrm{~mJ})$ corresponding to a peak pump fluence of $.8 \mathrm{~J} / \mathrm{cm}^{2}\left(1.3 \mathrm{~J} / \mathrm{cm}^{2}\right)$ for seeded (unseeded) operation. As expected, the unseeded OPO threshold is higher than for seeded operation because the unseeded OPO signal and idler waves have to build up from vacuum noise. The seeded OPO linewidth is approximately $150 \mathrm{MHz}$ while the unseeded linewidth is about $2-3 \mathrm{~cm}^{-1}$. The signal pulse has a duration of 
$6.5 \mathrm{nsec}$ FWHM at a pump encrgy of $11 \mathrm{~mJ}$. Both curves in Figure 2 show significant drop in slope efficiency with increasing pump energy. For seeded operation, the slope efficiency drops from 55\% near threshold to $26 \%$ for a pump energy of $11 \mathrm{~mJ}$. The conversion efficiency (signal energy divided by the incident pump energy) for signal wave production appears to saturate at $26 \%$ which is well below the theoretical quantum limit of $68 \%$ suggesting that only a fraction of the pump beam is depleted.

We have measured the time-integrated two-dimensional spatial profiles and the spatially-integrated timeresolved power of the incident and depleted pump beam and the signal beam. The depletion of the pump beam is consistent between the two measurements. Figures 3 and 4 show cross sections through the plane of the resonator of the time-integrated spatial profiles of the undepleted pump, depleted pump and the signal beam. The signal wave profile shown in Figure 3 has a Gaussian-like cross scation with a $.5 \mathrm{~mm}$ FWHM. This beam has been observed to be astigmatic with nearly diffraction limited divergence in the plane of the ring and greater divergence out of plane.

As shown in Fig. 4(a), the depleted pump profile at 3.1 times threshold $\left(2.5 \mathrm{~J} / \mathrm{cm}^{2}\right)$ shows local depletion as high as $75 \%$ and an overall energy reduction of $50 \%$. The depleted pump also shows an asymmetry in the svalkoff (horizontal) plane, i.e. more depletion is observed on the side of the beam toward which walkoff occurs. Note also that the maximum pump depletion does not occur at the peak incident pump position; an indication of back conversion, conversion signal and idler to pump, occurring in the center portion of the pump beam. Profiles at 1.1 times threshold $\left(.9 \mathrm{~J} / \mathrm{cm}^{2}\right)$, shown in Figure 4(b), also show significant pump depletion (20\% in energy). Note that even at this low pump fluence that the maximum depletion still occurs in the spatial wings of the pump beam; evidence that back conversion is occurring at the center of the pump beam.

In conclusion, we have carefully characterized the operation of a ring OPO. Spatial profiles show evidence of back conversion and asymmetries due to signal walkoff. This data will be useful in assessing the accuracy of transient 2-dimensional OPO models ${ }^{2}$.

This work was supported by the U.S. Department of Energy under Contract DE-AC04-76dp00789.

1. C.E. Hamilton and W.R. Bosenberg, in 1992 Conference on Lasers and Electro-Optics, 1992 OSA Technical Digest Series Vol. 12 (Optical Society of America, Washington, DC, 1992), p. 370.

2. M. Bowers and A.V. Smith, submitted to 1994 Advanced Solid-State Laser Conference.

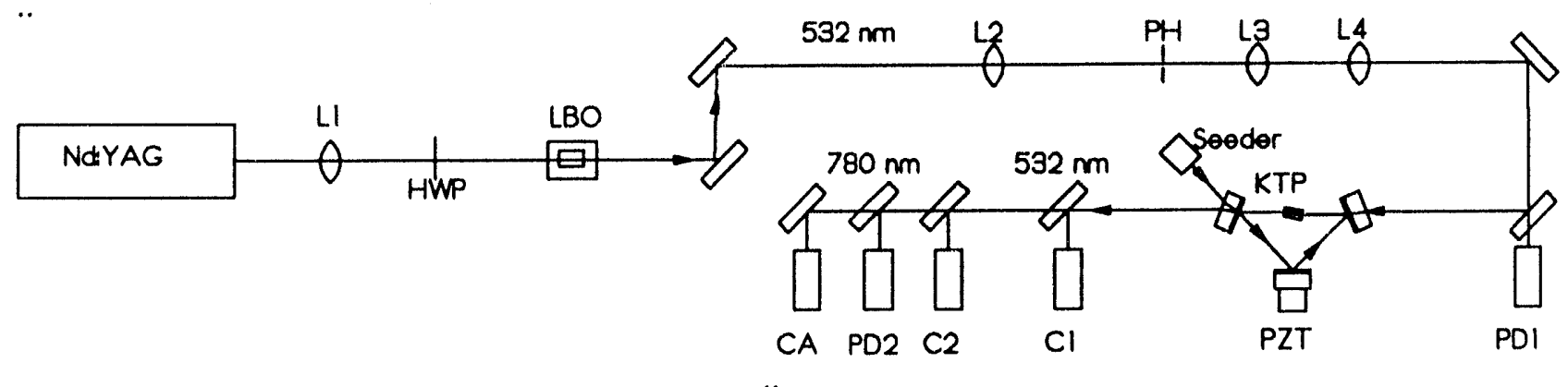

Figure 1 The experimental layout is shown above. Lens L1 weakly focuses the $1.06 \mu \mathrm{m}$ beam through the LBO crystal. A half wave plate (HWP) was used to vary the $532 \mathrm{~nm}$ energy produced in the LBO crystal. Lenses L2L4 and the pin hole (PH) spatially filter and collimate the green beam. Photodiode PD1 monitors the pump energy incident on the OPO. Cameras $\mathrm{Cl}$ and $\mathrm{C} 2$ monitor the green and signal beam profiles, photodiode PD2 monitors the signal temporal profile and pyroelectric detector CA monitors the signal energy. 


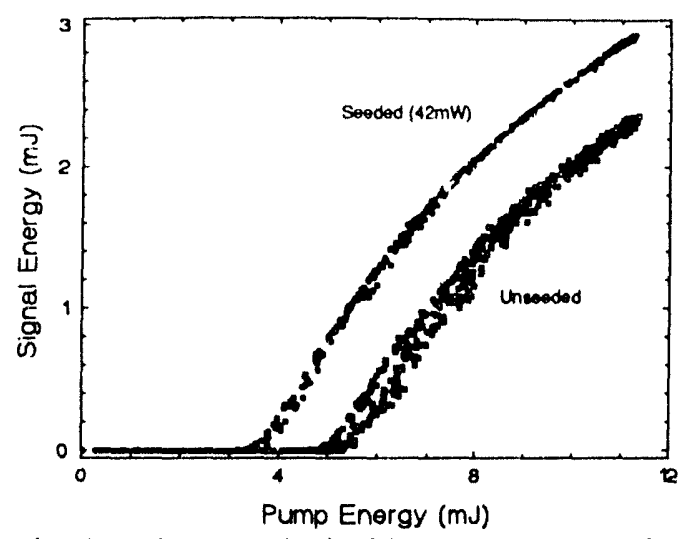

Figure 2 The output signal energy is plotted versus the incident pump energy for both seeded and unseeded operation. When seeded the slope of the output is 0.54 near threshold and the conversion efficiency appears to approach $26 \%$ at the maximum pump energy of $11 \mathrm{~mJ}$.

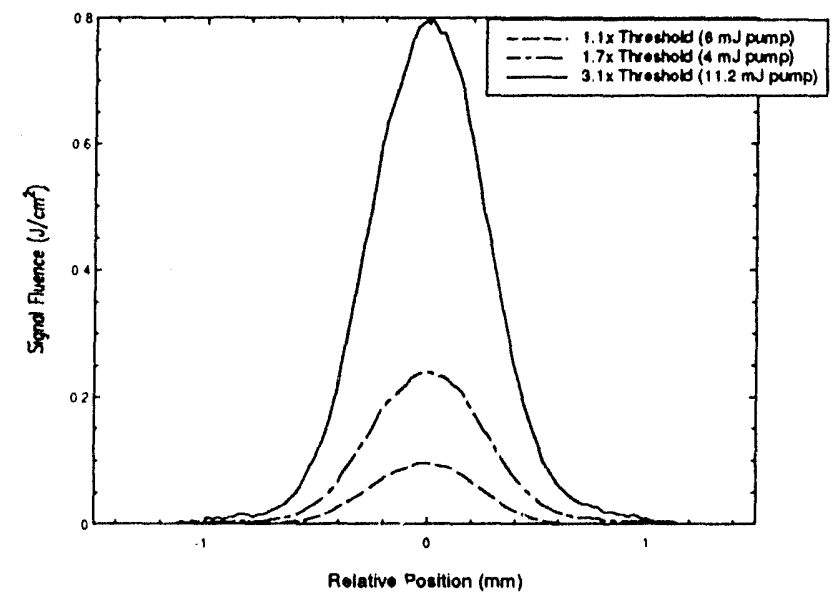

Figure 3 Profiles of the signal beam cut through ine plane of the resonator. The time integrated profiles of the signal beam remain smooth and Gaussian-like for the full range of pump fluences used.

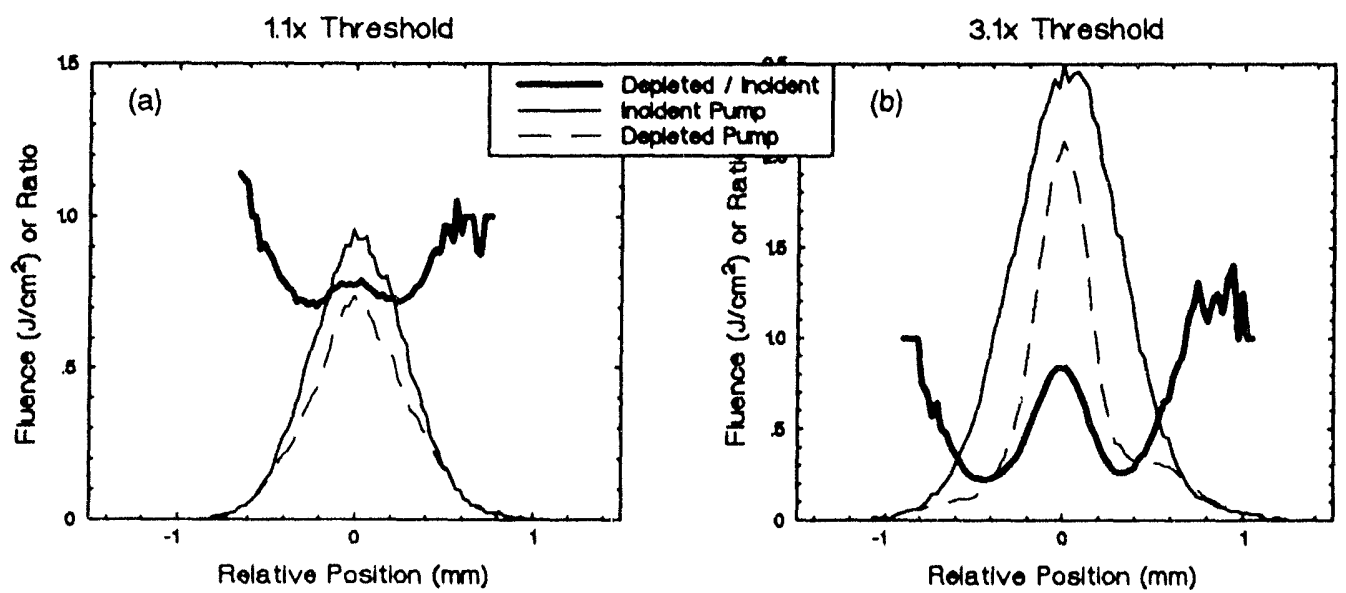

Figure 4 Profiles of the incident and depleted pump beam, cut through the plane of the resonator, are plotted above for pump encrgies of $4 \mathrm{~mJ}(1.1 \times$ threshold $)$ and $11.2 \mathrm{~mJ}(3.1 \times$ threshold $)$. Also plotted are the ratios of the depleted and incident profiles. Note that the maximum depletion of the incident pump beam occurs in the wings of the profile and that little conversion occurs near the beam center. We believe this to be evidence of back conversion of the signal and idler beams to pump. 
